\title{
Persistence of the SCR following few Pavlovian acquisition trials: An instrumental reinforcement process
}

\author{
ROBERT J. McCAFFREY \\ Alderson-Broaddus College, Philippi, West Virginia 26416
}

and

\author{
AVRUM I. SILVER \\ Medical University of South Carolina, Charleston, South Carolina 29401
}

\begin{abstract}
Classical conditioning of the SCR results in CR magnitude reaching a peak early in acquisition followed by attentuation of the CR. An inverse relationship has been demonstrated between the number of postpeak acquisition trials $(2,4,8,16)$ and subsequent resistance to extinction. A possible explanation for these unusual results involves an instrumental reinforcement process. Specifically, removing the UCS shortly after the peak CR may instrumentally reinforce the occurrence of greater CR amplitude, whereas continued UCS presentations (acquisition trials) that result in attentuation may indicate a form of punishment for large CR magnitude. The present study tested this hypothesis by manipulating UCS intensity beginning with the third trial beyond the peak CR. Generally, the results indicated that most subjects demonstrated an inverse relationship between amplitude of the CR and the direction of the change in UCS intensity. The proposed instrumental reinforcement process was the most logical explanation of the data.
\end{abstract}

Classical conditioning of the skin-conductance response (SCR) appears to have a unique set of characteristics not common to classically conditioned skeletal responses. During conditioning, the magnitude of the SCR reaches maximal CR strength within two or three acquisition trials and then declines in strength (Campbell, 1936; Kimmel, 1966). The rapid increase in CR magnitude and subsequent decline was investigated further by Silver and Kimmel (1969). In that study, the number of acquisition trials beyond the maximum, or peak $\mathrm{CR}$, was examined to determine the effect of postpeak (PP) acquisition trials on the resistance to extinction of the CR. Subjects were given $1,2,4,8$, or 16 paired trials beyond the peak CR, followed by extinction. Generally, the results showed that the greater the number of PP acquisition trials, the weaker the resistance of the CR to extinction. Maximum resistance to extinction was achieved with only two PP acquisition trials. Since only two or three trials were required to reach the peak CR, a total of four or five paired trials produced the strongest resistance to extinction. The weakest resistance to extinction occurred with 16 PP trials. These findings were replicated by Schramm and Kimmel (1970).

Another indication of CR persistence is the number of trials in which a subject continues to respond beyond the peak CR. Rather than focusing only on resistance to

Address reprint requests to Robert J. McCaffrey, Department of Psychology, Alderson-Broaddus College, Philippi, West Virginia 26416. extinction, examining the total number of PP trials (acquisition and extinction) permits a more comprehensive picture of $\mathrm{CR}$ strength and also reveals the amount of time a subject continues to respond beyond the peak CR. An examination of the groups that received 2 and 16 PP paired trials in the Silver and Kimmel (1969) study reveals that, on the average, the 2-PP group required 43 trials to reach the extinction criterion, whereas the 16-PP group required only 23 trials beyond the peak CR to reach extinction. Schramm and Kimmel's (1970) replication produced similar results. Their 2-PP paired group continued to respond for 43 trials, compared with 22 trials for the 16-PP paired condition. While attenuation of the CR occurs during both PP acquisition and extinction, one might expect extinction trials, and not acquisition trials, beyond the peak CR to enhance attenuation and result in weaker CRs. The results of the Schramm and Kimmel (1970) and Silver and Kimmel (1969) studies indicate the opposite. Beginning extinction following 2 PP paired trials resulted in greater persistence of the CR than initiating extinction following $16 \mathrm{PP}$ paired trials. There were approximately four or five acquisition trials in the 2-PP paired condition, compared with 18 or 19 acquisition trials in the 16-PP paired condition. Since the former, and not the latter, produces strong persistence of the $\mathrm{CR}$, an explanation is in order.

One possibility is that the strength of the PP CR in the 2-PP paired condition is due to an instrumental process that is initiated when the extinction procedure 
begins following 2 PP paired trials. Essentially, what is being proposed is that reinforcement of large CRs takes place when the shock UCS is removed following the peak CR. The persistence of the CR during extinction is reinforced by the continued absence of the UCS. This reinforcement process, which is not present when acquisition trials continue beyond the peak $\mathrm{CR}$, may be responsible for the persistance of the CR in the 2-PP condition.

To test this hypothesis, subjects in the present experiment received paired presentations of the CS and UCS until a criterion of 2 PP CRs was obtained. The subjects were then assigned to one of three UCS intensity conditions. Condition 1 involved an increase in the intensity of the UCS, Condition 2 involved maintaining the same UCS intensity, and Condition 3 consisted of a decrease in the UCS intensity. If an instrumental reinforcement process is operating in the 2-PP condition, then the decreased UCS intensity group should produce the strongest CRs, and increasing UCS intensity should result in weaker CRs. Maintaining the same UCS intensity should result in a level of CR strength that is intermediate in relationship to the other two conditions.

\section{METHOD}

\section{Subjects and Design}

Ninety-six undergraduate students from introductory psychology classes served as subjects. The subjects were randomly assigned to one of six equal groups of 16 subjects, with the restriction that the sexes be equally represented in each of the groups.

Three of the groups received paired trials up to the peak and were given two additional paired trials beyond the peak. Paired CS-UCS presentations continued for four more trials, with the groups receiving an increase, a decrease, or no change in UCS intensity beginning with the third paired trial beyond the peak. Subjects in three control groups were matched as to the number of acquisition trials, but they received CS-UCS presentations in an unpaired and random manner. The trial on which the intensity of the UCS was decreased, increased, or unchanged was also matched to the experimental groups, and the CSs and UCSs continued to be presented but unpaired.

\section{Apparatus}

Subjects were conditioned in a dark soundproof I.A.C. chamber. Each subject was seated in an armchair, and an intercom was used to communicate with the subject. The CS was a 39-dB (re: .0002 dynes $/ \mathrm{cm}^{2}$ ) 1,000-Hz tone of $5.0 \mathrm{sec}$ duration. The UCS was a $3.0-\mathrm{mA}$ dc electric shock with a duration of $.1 \mathrm{sec}$ produced by an Argonaut Associated LRA 046 constantcurrent pulse generator. The electric shock was delivered to the volar surface of the subject's right forearm.

The skin-resistance response (SRR) was picked up from the volar and palmar surfaces of the subject's left hand by zinc/ zinc sulfate electrodes. Skin-resistance changes were amplified by an Electronics Laboratory, Inc., skin-resistance amplifier (Model 308C) and were recorded on a Texas Instruments ReitiRiter with a paper speed of $15.5 \mathrm{~cm} / \mathrm{min}$.

\section{Procedure}

The subjects were seated in the chamber with the lights turned off and the doors closed. A set of instructions was read that asked them to remain as still as possible and to avoid deep breathing or sleeping. After 2 min of adaptation, all groups received three presentations of the UCS alone in increasing intensities of $1.0,2.0$, and $3.0 \mathrm{~mA}$. Then all groups received habituation trials or 12 presentations of the CS only (Silver, 1973). The experimental subjects received paired presentations of the CS and UCS until they reached a criterion of two paired trials past the peak CR. On the third trial past the peak, a change in intensity took place for some subjects.

To ensure that the change was subjectively equidistant from the initial 3.0-mA shock, a procedure outlined by Stevens, Carton, and Shickman (1958) was employed. The procedure was used with 10 subjects who were not included in the current study. The values that were judged as being an equal distance in apparent intensity from $3.0 \mathrm{~mA}$ were $4.8 \mathrm{~mA}$ for the increase and $1.0 \mathrm{~mA}$ for the decrease. Therefore, on the third trial beyond the peak for the paired groups, UCS intensity increased to 4.8 , decreased to 1.0 , or maintained at $3.0 \mathrm{~mA}$ for the following four paired trials. The same occurred for the three control groups, in that they were matched to the paired groups for number of trials to the peak and the subsequent intensity change.

A delayed conditioning paradigm was employed with a 5 -sec interstimulus interval and a 20 to 50 - sec interstimulus interval that varied around a mean of $35 \mathrm{sec}$. When the experiment was over, each subject was debriefed, thanked, and dismissed.

\section{RESULTS}

For the purposes of statistical analysis, the CR magnitudes in units of resistance were transformed into conductance units via the formula: $\log C[(\mathrm{SRL}-\mathrm{SRR}) /$ $\mathrm{SRL}]^{-1}$. The CR(SRR) was defined as a decrease in resistance greater than $1.0 \%$ of the value of the skinresistance level (SRL) $1.0 \mathrm{sec}$ prior to the occurrence of a CR. For a response to be called a CR, it had to occur within 1.0-2.5 sec from the onset of the CS.

The test for sampling differences and conditioning involved only the first four acquisition trials, for to go beyond would include differences in UCS intensity. To test for sampling differences during the first four acquisition trials, two separate groups by conditions by trials analyses of variance were performed: one for the paired conditions and the other for the unpaired groups. The outcome of these analyses revealed that there were no statistically significant differences within the paired or unpaired conditions. To test for conditioning, all paired groups were combined and all unpaired groups were combined. A groups by trials analysis of variance on these measures for the first four acquisition trials revealed a main effect of pairing $[F(1,30)=20.60]$ and trials $[\mathrm{F}(3,90)=12.85]$, as well as the Pairing by Trials interaction $[\mathrm{F}(3,90)=18.81]$. All three were significant $(p<.001)$, demonstrating that conditioning had occurred.

The effects of the changes in UCS intensity on mean CR magnitude are presented in Figure 1. A pairing by intensity by trials analysis of variance revealed significant effects of pairing $[\mathrm{F}(1,90)=29.31, \mathrm{p}<.001]$, trials $[\mathrm{F}(3,270)=3.12, \mathrm{p}<.025]$, and Trials by Intensity $[F(6,270)=2.88, p<.01]$. Nonetheless, the data contain several interesting trends that suggest a different mode of analysis. The paired group that received the decrease in UCS intensity showed little or no CR attenu- 
ation, whereas the group that received the increase in UCS intensity appeared to demonstrate CR attenuation. However, the nature of the dependent variable in Figure 1, mean CR magnitude, possesses characteristics that might have obscured the hypothesized effects.

The CR magnitudes in Figure 1 reflect an average in the responsivity of a group of subjects on a specific trial, and therefore they are sensitive to extreme scores. For example, the majority of subjects on a specific trial might show a moderate increase in CR amplitude over the previous trial, and the remaining one or two subjects might demonstrate large decreases in CR amplitude in comparison with the previous trial, the result being little or no change in CR magnitude for that trial in comparison with the preceding trial. Extreme increases or decreases in amplitude by one or two subjects in the experimental conditions could effectively mask any small, yet systematic, within-subjects changes in CR amplitude. An examination of the number of subjects who showed an increase in CR amplitude from one trial to the next might provide information that would otherwise be obscured by the more traditional CR magnitude measures. Therefore, an additional analysis was performed on the amplitude data, beginning with the trial on which a change in UCS intensity took place.

This analysis consisted of trial-to-trial comparisons for each subject. The CR amplitude on the third trial past the peak (i.e., the trial on which the UCS intensity changed) was compared with the CR amplitude on the fourth trial past the peak, then the fourth with the fifth, and the fifth with the sixth. If the amplitude of the second CR was greater than the amplitude of the first $\mathrm{CR}$, then that comparison was scored as a " 1 "; if the comparison was less than or equal to, then it was scored as a "0." The total number of increases within each of these comparisons was then summed across subjects, and that sum was converted to a percentage score. These

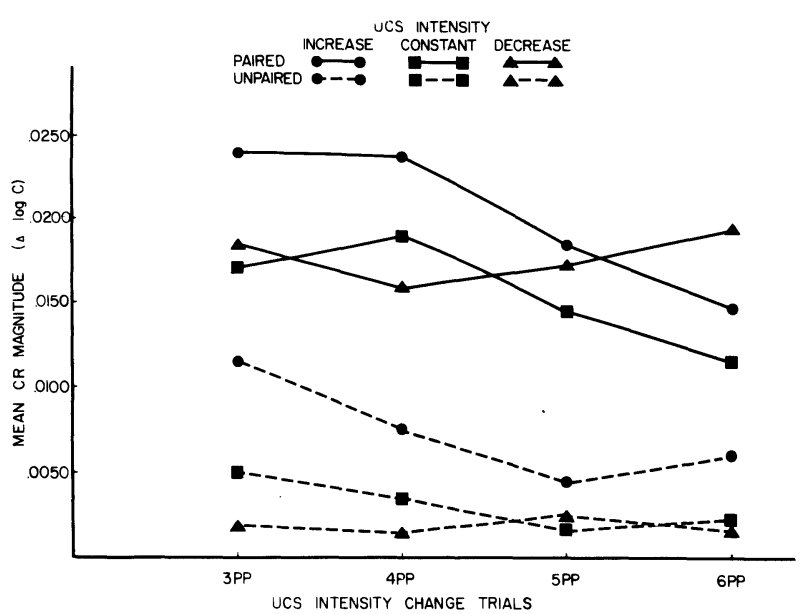

Figure 1. Mean CR magnitude following a change in UCS intensity for paired and unpaired groups.

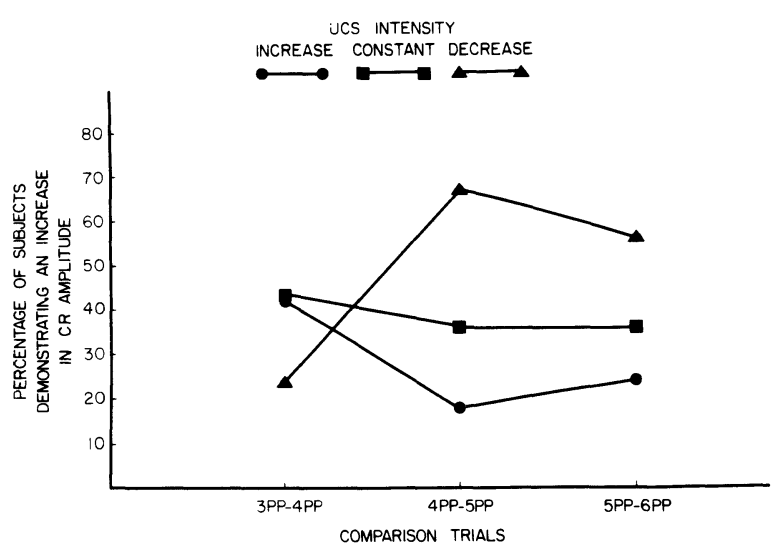

Figure 2. The percentage of subjects demonstrating an increase in CR amplitude on three comparison trials following the manipulation of UCS intensity in the paired CS-UCS groups.

data are presented in Figure 2 and represent the percentage of subjects who showed an increase in CR amplitude from one trial to the next.

Moving from the first comparison to the second comparison in Figure 2, more subjects in the decreased intensity group showed an increase in CR amplitude than in the constant or increased groups. In the decrease group, over $50 \%$ of the subjects on the second and third comparisons showed increases in CR amplitude, despite the fact that they experienced decreases in UCS intensity. Also, on the second and third comparisons, less than $30 \%$ of the subjects demonstrated increases in CR amplitude, even though they received an increase in UCS intensity. Most subjects receiving no change in UCS intensity showed decreases in CR amplitude for each of the three comparisons. Within the constant UCS intensity group, the percentage of subjects showing a decrease fell between the two other groups for both the second and third comparisons. The data were subjected to an analysis of variance following the recommendations of Gabrielsson and Seeger (1971) and Seeger and Gabrielsson (1968). The results of the analysis revealed a significant main effect of change in UCS intensity $[F(2,45)=4.0, p<.025]$. The Trials by Intensity interaction approached significance $[F(4,90)=2.0, p<.10]$.

\section{DISCUSSION}

The first few trials of SCR conditioning produce large CRs, which in previous studies (e.g., Schramm \& Kimmel, 1970; Silver \& Kimmel, 1969; Silver, McCaffrey, \& Godfrey, 1978) were followed by the omission of the UCS. The result has been strong persistence of the CR both in magnitude and in trials to the extinction criterion. The possibility that UCS omission reinforces large SCRs was tested and received tentative support in the present experiment.

The effects of a change in the UCS were examined through the use of a relatively novel technique. Following the peak CR, subjects received increases, decreases, or no change in UCS intensity. Generally, the data revealed that more subjects demon- 
strated increases in amplitude when the UCS intensity decreased. Conversely, with an increase in UCS intensity, most subjects demonstrated decreasing CR amplitudes from one trial to the next. This is unusual, since other studies have demonstrated a direct relationship between intensity and amplitude (e.g., Passey, 1948; Prokasy, Grant, \& Meyers, 1958; Silver et al., 1978). These unusual results appear to be best accounted for by an instrumental reinforcement explanation.

Additional support for this proposed process comes from the study by Silver et al. (1978). In that study, three intensity levels of the UCS, 1, 2, and $4 \mathrm{~mA}$, and two levels of PP acquisition trials (i.e., 2 and $16 \mathrm{PP}$ ) were employed. Based on these findings, it appears that the removal of the UCS results in a less effective instrumental reinforcing process when UCS intensity levels are below $4 \mathrm{~mA}$.

The present research has implications for instrumentally modifying autonomic responses. To date, much of the research has been concerned with using autonomic responses in an instrumental conditioning procedure (e.g., Katkin, 1968; Kimmel, 1974). However, the present study provides evidence for an instrumental process within a classical conditioning procedure. This process accounts for the results from a Pavlovian conditioning procedure that would be otherwise difficult to explain. It also contributes to the notion that organismic processes involved during classical conditioning of the SCR are rather cognitive in nature and more complicated than was originally thought.

Finally, since the SCR lends itself to the study of anxiety, the results of the current experiment have implications for the acquisition of anxiety (e.g., Silver, 1977). The notion that strong and effective acquisition of an autonomic response takes place following so few conditioning trials is interesting because it suggests that an emotional response can be acquired in very few trials. This strong and persistent reaction following so few conditioning trials might provide some explanation for the acquisition of very strong and highly persistent emotional responses such as phobias.

\section{REFERENCES}

Campbell, R. K. Some difficulties in conditioning galvanic skin responses. Psychological Bulletin, 1936, 33, 618-619.

Gabrielsson, A., \& Seeger, P. Tests of significance in twoway designs (mixed model) with dichotomous data. British Journal of Mathematical and Statistical Psychology, 1971, 24, 111-116.
Katkin, E. S., \& Murray, E. N. Instrumental conditioning of autonomically mediated behavior: Theoretical and methodological issues. Psychological Bulletin, 1968, 70, 52-68.

KIMMEL, H. D. Inhibition of the unconditioned response in classical conditioning. Psychological Review, 1966, 73, 232-240.

KIMMEL, H. D. Instrumental conditioning of autonomically mediated responses in human beings. American Psychologist, 1974, 29, 325-335.

Passey, G. E. The influence of intensity of unconditioned stimulus upon acquisition of a conditioned response. Journal of Experimental Psychology, 1948, 38, 420-428.

Prokasy, W. F., Grant, D. A., \& Meyers, N. A. Eyelid conditioning as a function of unconditioned stimulus intensity and intertrial interval. Journal of Experimental Psychology, $1958,55,242-246$.

Schramm, C. F., \& Kimmel, H. D. Resistance to extinction in GSR conditioning following different number of postpeak acquisition trials. Journal of Experimental Psychology, 1970, 84, 239-243.

Seeger, P., \& Gabrielsson, A. Applicability of the Cochran $\mathrm{Q}$ test and the $\mathrm{F}$ test for statistical analysis of dichotomous data for dependent samples. Psychological Bulletin, 1968, 69, 269-277.

Silver, A. I. Effects of prior CS presentations of classical conditioning of the SCR. Psychophysiology, 1973, 10, 583-588.

Silver, A. I. Recent developments in classical conditioning of the galvanic skin response: Implications for the acquisition of anxiety. Journal of Behavior Therapy and Experimental Psychiatry, 1977, 8, 337-338.

Silver, A. I., \& Kimmel, H. D. Resistance to extinction in classical GSR conditioning as a function of acquisition trials beyond peak CR size. Psychonomic Science, 1969, 14, 53-55.

Silver, A. I., McCaffrey, R. J., \& Godfrey, G. Effects of UCS intensity and postpeak acquisition trials on classical conditioning of the SCR. Acta Psychologica, 1978, 42, 225-230.

Stevens, S. C., Carton, A. S., \& Shickman, G. M. A scale of apparent intensity of electric shock. Journal of Experimental Psychology, 1958, 56, 328-334.

\section{NOTE}

1. There were only five comparisons in which the amplitude of the CRs were equal to each other, one in the increased condition, one in the decrease condition, and three in the constant condition.

(Received for publication March 8, 1980.) 